Antwerpen: Gerard Leeu, 1486. Titel (in Kolophon): Presens liber Dyalogus creaturarum appellatus... HC 6129.

Antwerpen: Gerard Leeu, 1491. Titel (in Kolophon): Presens liber Dyalogus creaturarum appellatus... HC 6129.

Destructorium vitiorum ex similitudine creaturarum exemplorum appropriatione per modum dialogi, [Genève: Jean Bellot], 1500. H 6131, HC 654.

Dialogus creaturarum optime moralisatus, Köln: Retro Minores (Martin von Werden), [ca. 1505]. C 1956, VD 16 N 1562.

Destructorium vitiorum ex similitudine creaturarum exemplorum appropriatione per modum dialogi, Lyon: Claude Nourry, 1509.

Dialogus creaturarum optime moralizatus..., Paris: Philippe Pigouchet für Jehan Petit, 1510.

Destructorium vitiorum ex similitudine creaturarum exemplorum appropriatione per modum dialogi, Lyon: Claude Nourry, 1511.

\title{
R49. Anonymus
}

\section{Liber contra Iudaeos}

Pharetra contra Iudaeos / Tractatus disputans contra Iudaeos / Tractatus de Iudaeis / Liber Talmoth seu altercationes contra Iudaeos / Pharetra fidei catholicae

Studie: Kapitel 3.2.

Titel: In der Praefatio nennt sich der Text Liber contra Iudaeos (siehe Explicit des Prologs), in der Hamburger Handschrift mit dem Zusatz seu appellatur Pharetra Iudeorum. Pharetra contra Judaeos wird als Titel in der Frankfurter Handschrift angegeben, Liber Talmoth in der Tübinger, während die Drucke sich mehrheitlich für Pharetra fidei catholicae sive ydonea disputatio entscheiden. Der Aufsatz Cardelle de Hartmann, „Drei Schriften“, nennt den Text „Pharetra 3“ zur Unterscheidung von anderen antijüdischen Schriften mit diesem Titel (siehe R26).

Prolog: In den Handschriften: Incipit: Scimus, fratres, quod diversa opinio ex Iudaeis... Explicit:... Intituletur autem iste liber contra Iudaeos quia firmiter agit contra eos. [Varianten: in einigen Hss. fratres karissimi statt fratres; Hs. München, Clm 23786: intelligatur statt intituletur; Hs. München, Clm 3762: quia...eos fehlt; Hs. Hamburg fügt am Ende hinzu: seu appellatur Pharetra Iudaeorum].

In den Drucken: Incipit: Ad Iudaeorum confutationem omnis Christi fidelis tollat pharetram hanc et arcum... Explicit:... Alia persona est loco christiani qui modo subtiliori procedit per dicta prophetica et sanctorum patrum. Das Vorwort in 
den Drucken besteht aus Sätzen des Prologs in den Handschriften und Sätzen des Prologs zu der Pharetra des Thibaut de Sézanne (Theobaldus de Sexannia) - zu diesem Werk vgl. Eintrag „Nicolaus de Argentina Autor" und Cardelle de Hartmann, „Drei Schriften“, 328-335.

Incipit: Iudaeus dicit: Vos christiani credentes dicitis temere Deum esse trinum... [Hss. Frankfurt und Hamburg]; Iudaeus dicit: Vos christiani temere creditis quod dicitis Deum esse trinum... [Hss. München, Clm 3762, und Tübingen]; De trinitate Iudaeus arguit: Vos christiani tenore [sic] creditis dicentes Deum esse trinum... [Hs. München, Clm 23786]; Iudaeus dicit: Vos christiani credentes temere dicitis Deum esse trinum... [Drucke].

Explicit: Iudaeus dicit: convertat nos Christus. Respondit christianus: Amen [Hss. Frankfurt, München, Clm 23786, und Hamburg; Variante in der Hs. München, Clm 23786: Deus statt Christus]; Iudaeus dicit: convertat nos Deus christianorum. Amen [Hs. München, Clm 23786]; Tunc dixit Cristianus. Amen [Hs. Tübingen]; Iudaeus respondit ultimo: conducat nos per viam iustitiae ut veritatem valeamus percipere ab eo qui est benedictus in saecula saeculorum. Amen [Drucke].

Autor: Die Überlieferung legt nahe, dass der Autor Deutscher war. Diese Vermutung sieht sich durch eine Passage erhärtet, in der das deutsche Wort Endechrist (Antichrist) als „Ende der Christenheit“" gedeutet wird. Der Text lautet in der Hs. Frankfurt, fol. 44va: Vos forsitan nominatis antichristum in aduentum antichristi et ille erit uerus iudex inter nos et uos quia eo adueniente exter < min>abitur christianitas et ergo uocatur in almanico endecrist quasi finis christianitatis. Andere Handschriften lesen theutonice statt almanice und Enchrist (Hs. Tübingen) oder Anterchrist (Hss. München) statt Endechrist. Auch die unter „Datierung“ erwähnte Textstelle passt zu einem deutschen Autor. Diese Stelle fehlt in den Drucken.

Der Dialog wird in Clm 23786 mit einer Sammlung von kleinen Schriften des Augustinus überliefert, was den Schreiber wohl zur Zuschreibung an Augustinus verleitet hat.

Fassungen: Es gibt auffallende Unterschiede zwischen dem Text in den Handschriften und in den Drucken. Inhalt und Umfang sind weitgehend gleich, mit folgenden Ausnahmen: Im Druck wurde der erste Teil der Diskussion (Dreifaltigkeit) gekürzt, die Prophezeiung über das Kommen des Messias (siehe unter „Datierung ${ }^{(6)}$ und die kurze Erklärung über den Antichrist ganz weggelassen. Hinzugefügt wurde eine lange Passage (zwei Seiten in den Drucken, quarto-Format) am Ende, die eindeutig aus der Errores fudeorum in Talmut genannten Sammlung 
von Talmudexzerpten (siehe R26 - Autor und Cardelle de Hartmann, „Drei Schriften“, 328-335) genommen wurde. Es handelt sich um Textstellen, in denen geschildert wird, dass die Juden die Christen belügen und betrügen dürfen und jeden Tag um den Untergang der christlichen Reiche beten. Im Druck ist die Sprache grammatikalisch und stilistisch geglättet, einige Wiederholungen sind weggelassen. Der Ton der Diskussion wird aber eindeutig verschärft, fast jeder Beitrag wird mit einer Beleidigung eingeleitet und einige Behauptungen, die in den Handschriften allgemein aufgestellt werden, werden im Druck personalisiert und auf den mitdiskutierenden Juden bezogen. In den Drucken fehlt jeder Hinweis auf den Redaktor oder Herausgeber des Textes.

Der Liber war außerdem mit großer Wahrscheinlichkeit die Vorlage für den Tractatus contra fudaeos des Petrus de Pennis OP (vgl. den Eintrag R59 „Petrus de Pennis“).

Datierung: Eine Stelle in den Handschriften gibt einen Anhaltspunkt für die Chronologie. Der Jude erwähnt eine Berechnungsmethode für den Zeitpunkt der Ankunft des Messias, die sich auf eine Chronologie deutscher Kaiser stützt. Die Stelle lautet in der Frankfurter Handschrift: Fudeus: Nos habemus unum verbum in Fudaico quod dicitur alcom, in illo verbo sunt descripti omnes Cesares Romanorum, per aleph $\boldsymbol{\aleph}$ intelligitur Albertus cesar primus regnans, per lameth $ל$ Lodizercus, per vau $ר$ Vredericus, per coph $>$ Conradus, per mem $\square$ Messias. Sed ultimus fuit Vredericus, post hunc Messias non fuit visus. (fol. 44va, in der Handschrift sind die hebräischen Buchstaben leicht entstellt). Die Interpretation dieser konfusen Stelle ist schwierig. Wenn wir unter cesares nicht nur Kaiser, sondern auch deutsche Könige verstehen, kommen wohl in Frage Albrecht I. (1298-1308), Ludwig der Bayer (1314-1347) und Konrad IV. (1250-1254). Die Identifizierung des genannten Friedrich ist hingegen problematisch. Ohne die Bemerkung, er sei der letzte gewesen, käme Friedrich II., der „Endzeitkaiser“ schlechthin, in Frage. Wenn wir nach jüngeren Trägern des Namens suchen, bieten sich zwei Staufer mit dem Namen Friedrich an, die wegen Name und Abstammung als „dritter Friedrich“ angesehen werden konnten: Friedrich der Freidige (1257-1323), Markgraf von Meißen und Landgraf von Thüringen, und Friedrich II. (III.) von Aragón, König von Sizilien (ca. 1272-1337). Allerdings war keiner der beiden römischer Kaiser oder deutscher König. Möglich wäre noch der Gegenkönig Friedrich der Schöne (1314-1330). Da die (datierte) Frankfurter Handschrift Mitte des 14. Jhs. entstanden ist, kommen 
spätere Träger des Namens nach Friedrich dem Schönen nicht mehr in Frage. Aber auch bei den drei Friedrichen aus dem 14. Jh. stehen wir vor dem Problem, dass sie alle vor Ludwig dem Bayern gestorben sind, dass daher keiner von ihnen als ultimus bezeichnet werden konnte. Eine überzeugende Identifizierung scheint nicht möglich. Der Text dürfte etwa in das zweite Viertel des 14. Jhs. zu datieren sein, nicht viel früher als die Frankfurter Handschrift.

Inhalt: Gespräch zwischen Judaeus und Christianus, ohne Einleitung oder narrativen Rahmen. Die verschiedenen Themen werden mit einem Redebeitrag des Juden eingeleitet, in dem er Einwände gegen einen Bereich des christlichen Glaubens vorbringt. Der Christ erklärt daraufhin den christlichen Glauben mit einfachen Worten, wobei er sich auf Argumente aus der Natur und auf Zitate aus der Bibel, vor allem von den Propheten, stützt. Er verwendet weder Vernunftargumente noch dialektisch aufgebaute Beweise. Zum Teil werden die Stellen aus der Schrift allegorisch oder typologisch gedeutet. Der Jude argumentiert nicht dagegen, sondern fragt nach weiteren Aspekten oder bringt neue Einwände vor. Er bezieht sich mitunter auf das Neue Testament oder das christliche Glaubensbekenntnis. Die Redebeiträge des Juden sind meistens sehr kurz, etwas ausführlicher nur dann, wenn er ein neues Thema einführt. Der Christ hat zwar größere Redeanteile, seine Antworten werden trotzdem ziemlich knapp gehalten.

Das erste Thema, das der Jude anspricht, ist die Dreifaltigkeit. Er wirft dem Christen vor, mehrere Götter anzubeten, und betont die Widersprüchlichkeit der christlichen Lehre. Der Christ erklärt die christliche Doktrin mit Hilfe von Beispielen aus der Natur und behauptet, man müsse daran glauben, weil der christliche Glaube der echte und wahre sei. Der Jude geht dann auf die Jungfräulichkeit Mariens ein, die der Christ mit Textstellen aus den Propheten verteidigt. Das nächste Thema ist die Menschwerdung Gottes, deren Sinn und Notwendigkeit der Jude anzweifelt. Der Christ argumentiert wieder mit Bibelstellen, ohne auf die theologische Diskussion oder auf die Rechtfertigung der Inkarnation einzugehen. Es folgt ein abrupter Themenwechsel, als der Jude den Christen vorwirft, sich nicht beschneiden zu lassen und somit das Gesetz zu missachten. Der Christ ist zwar bereit, der Beschneidung eine gewisse Wirkung zuzusprechen, diese sei jedoch nicht mit der Wirkung der Taufe zu vergleichen. Die Taufe habe durch ihre gnadenspendende Kraft die Beschneidung überflüssig gemacht. Der Jude fragt anschließend nach dem Sinn der Kreuzigung. Der Christ antwortet 
mit einer typologischen und allegorischen Interpretation des Kreuzes und anderer Leidensmerkmale. Der Jude behauptet nun, Jesus sei nicht der Messias, worauf der Christ mit einer allegorischen Deutung der messianischen Prophezeiungen antwortet. In den Handschriften wird anschließend das Kommen des Antichrist angesprochen. Der Jude interpretiert den deutschen Namen Endechrist als „Ende der Christenheit“ und denkt deshalb, der Antichrist werde der Richter zwischen Juden und Christen sein und zugunsten der Juden urteilen. Der Christ erklärt ihm dann, dass Antichrist aus dem Griechischen komme und den Gegner Christi bezeichne. Mit seinem Kommen soll er die Christen prüfen. Nur ganz kurz wird der nächste Vorwurf des Juden behandelt, dass die Christen Bilder anbeten. Der Christ kontert mit der Behauptung, Gott liebe die Juden nicht mehr, was man an den Leiden dieses Volkes sehen könne. Der Christ wirft dem Juden ebenfalls vor, der Talmud sei häretisch (allerdings, ohne auf bestimmte Inhalte einzugehen), und bespricht die Nahrungsgesetze der Juden, die er als Strafe sieht. Der Jude betont seinerseits die Gesetzesuntreue der Christen, die den Sabbath, ja, sogar den Sonntag, brechen. Darauf erwidert der Christ nur, dass jeder sich für seine Handlungen werde verantworten müssen. Das nächste Thema ist die Eucharistie. Der Jude behauptet, es sei unmöglich, dass Gott in Brot gegenwärtig sei. Der Christ erklärt seinen Glauben auf einfachste Weise. In den Drucken schließt sich eine lange Rede des Christen an, in der er ausbreitet, wie der Talmud den Hass der Juden gegen die Christen festschreibe. Er zitiert sogar einen Fluch gegen die Christen, der sich im Talmud befinden soll. Am Schluß äußert der Jude unvermittelt seinen Willen zur Bekehrung.

\section{Editionen:}

Unediert.

Eine kritische Edition wird von Carmen Cardelle de Hartmann geplant.

\section{Literatur:}

Cardelle de Hartmann, Carmen, „Drei Schriften mit dem Titel Pharetra fidei“, Ashkenas 11 (2001), 327-349.

Schreckenberg, Heinz, Die christlichen Adversus-Fudaeos-Texte und ihr literarisches und historisches Umfeld (13.-20. Jh.), Frankfurt 1994, 565-566 (der Autor kennt nur die Drucke und datiert das Werk deswegen in das 15. Jh).

\section{Überlieferung:}

Handschriften:

Aus In principio:

Frankfurt a. M., StUB, Ms. Barth. 163, 14. Jh. (Mitte), fol. 38r-47r. Titel: Pharita [sic] contra Iudeos. Konsultiert in Reproduktion (Reader-PrinterKopien). 
München, BSB, Clm 23786, a. 1469, fol. 151r-163r. Titel: Tractatus Sancti Augustini in quo disputat contra perfidos Judaeos. Kolophon: Explicit tractatus sancti Augustini disputans contra Fudaeos. Autopsie.

Tübingen, UB, Mc 149, 15. Jh. (Anfang), fol. 158r-171v. Titel: Liber Talmoth seu Altercationes contra Fudaeos. Konsultiert in Reproduktion (Reader-PrinterKopien).

Hinzu kommen:

Halle an der Saale, ULB Sachsen-Anhalt, Qu. Cod. 104, a. 1383, fol. 136r-157r. Die Handschrift wird in SOPMA unter dem Tractatus des Petrus de Pennis aufgeführt. Sie überliefert den Prolog des Petrus, allerdings vermerkt der Katalog im Dialog Unterschiede zum Text des Petrus de Pennis in anderen Handschriften. Das im Katalog angegebene Explicit stimmt mit dem des Liber überein. Der Text ist im Kolophon datiert: Expliciunt dicta et signacula talomot quem fudei reputant pro magno doctore in lege doctorum eorum. Ano [sic] domini MCCCLXXXIII in vigilia Pasche per manus domini Iohannis Bussen plebani in Sman.

Hamburg, SUB, theol. 1534, 15. Jh. (hier 1424), fol. 249va-261ra. Ohne Titel. In dieser Handschrift findet sich nicht derselbe Prolog wie in der restlichen Überlieferung, sondern der Prolog zum Tractatus contra Iudaeos des Petrus de Pennis (siehe R59). Konsultiert in Reproduktionen (Mikrofiche).

München, BSB, Clm 3762, 15. Jh., fol. 251r-265v. Titel: Alter tractatus de Iudeis. Autopsie.

Frühdrucke:

Pharetra fidei contra iudeos, s.l. s.a. [Straßburg: Peter Attendorn, c. 1489-92]. H 12911, BSB-Ink. T-141.

Pharetra fidei catholice siue ydonea disputatio inter Christianos et Judeos, Leipzig: Konrad Kachelofen, 1495. H 12914, BSB-Ink. T-142.

Pharetra fidei catholice siue ydonea disputatio inter Christianos et Fudeos, Leipzig: Arnold von Köln, [c. 1494]. HC 12913, BSB-Ink. T-143.

Pharetra fidei catholice siue ydonea disputatio inter Christianos et fudeos, [Köln: Heinrich Quentell, c. 1494-1500]. HC 12912, BSB-Ink. T-144.

Pharetra fidei catholice siue ydonea disputatio inter Christianos et fudeos, Leipzig: Konrad Kachelofen, 1495. HC 12915, BSB-Ink. T-145. Nur geringfügige Unterschiede zu H 12914.

Pharetra fidei catholice siue ydonea disputatio inter Christianos et fudeos, Leipzig: Melchior Lotter, 1499. HC 12916, BSB-Ink. T-146.

Pharetra fidei catholice siue ydonea disputatio inter Christianos et fudeos, [Köln: Cornelius von Zierickzee, c. 1499]. HC 12910, BSB-Ink. T-147.

Pharetra catholice fidei sive ydonea disputatio inter Christianos et fudeos, Leipzig: Melchior Lotter, 1502. VD 16 P 2434.

Pharetra catholice fidei sive ydonea disputatio inter Christianos et Fudeos, Landshut: Johann Weyssenburger, 10. März 1514. VD 16 P 2435.

Pharetra catholice fidei sive ydonea disputatio inter Christianos et Fudeos, Landshut: Johann Weyssenburger, 15. April 1514. VD 16 P 2436.

Pharetra catholice fidei sive ydonea disputatio inter Christianos et Judeos, Landshut: Johann Weyssenburger, 1518. VD 16 P 2437. 